\title{
ANALYTIC DESCRIPTION OF THE PHASE TRANSITION OF INHOMOGENEOUS MULTIGRAPHS
}

\author{
ÉLIE DE PANAFIEU \\ Research Institute for Symbolic Computation (RISC) \\ Johannes Kepler Universität \\ Altenbergerstraße 69 \\ A-4040 Linz, Austria \\ VLADY RAVELOMANANA \\ Univ Paris Diderot, Sorbonne Paris Cité, \\ LIAFA, UMR 7089, \\ 75013, Paris, France
}

\begin{abstract}
We introduce a new model of random multigraphs with colored vertices and weighted edges. It is similar to the inhomogeneous random graph model of Söderberg [18, extended by Bollobás, Janson and Riordan [6]. By means of analytic combinatorics, we then analyze the birth of complex components, which are components with at least two cycles.

We apply those results to give a complete picture of the finite size scaling and the critical exponents associated to a rather broad family of decision problems. As applications, we derive new proofs of known results on the 2-colorability problem [16] and on the enumeration of properly $q$-colored multigraphs [19]. We also obtain new results on the phase transition of the satisfiability of quantified 2-Xor-formulas 8, 7.

Keywords. generating functions, analytic combinatorics, inhomogeneous graphs, phase transition
\end{abstract}

\section{INTRODUCTION}

Phase transitions for Boolean Satisfiability (SAT) and for Constraint Satisfaction Problems (CSP) are fundamental problems arising in different communities including computer science, mathematics and physics. For any $k \geq 2$, the random version of the well-known $k$-SAT problem is known to exhibit a sharp phase transition [13: as the density $c$ of clauses (where the number of clauses is $c$ times the number of variables) increases, the formula abruptly changes from being satisfiable to being unsatisfiable at a critical threshold point. For general CSP, the last decade has seen a growth of interest in determining the nature of the SAT/UNSAT phase

This work was partially founded by the ANR Boole, the ANR Magnum and the Austrian Science Fund (FWF) grant F5004.

This is the long version of the homonym paper accepted in the proceedings of Eurocomb 2013. 
transition (sharp or coarse), locating it, determining a precise scaling window and better understanding the structure of the space of solutions. These turn out to be very challenging tasks (see e.g. [9, [1).

1.1. Related Works. Recently, different authors gave precise descriptions of the phase transitions associated to several tractable decision problems.

The 2-colorability problem consists in determining if the vertices of a given graph can be colored with two colors in such a way that the vertices of each edge of the graphs have distinct colors. Pittel and Yeum [16] derived the limit probability for a random graph $G(n, m)$ with $n$ vertices and $m$ edges to be 2-colorable, when the parameter $m / n$ is smaller than or in the vicinity of $1 / 2$.

Almost at the same time, Daudé and Ravelomanana 8] considered the 2-Xor satisfiability problem in which each instance is a formula that is a conjunction of Boolean equations of the form $x \oplus y=0$ or $x \oplus y=1$. They showed that the probability that a random 2-Xor formula is satisfiable tends to a similar threshold function.

In [7, Creignou et al studied a quantified version of 2-Xor-SAT. They introduced $(a, 2)$-QXor formula, which are formula of the form $\forall X \exists Y \phi(X, Y)$ where $X$ and $Y$ denote distinct set of variables and $\phi$ is a Xor-formula with clauses containing exactly $a$ variables from $X$ and 2 variables from $Y$. The problem consists in determining if for every assignment of the variables $X$, there exists an assignment of the variables $Y$ such that $\phi(X, Y)$ is true. For any positive integers $a$, the authors showed that the phase transitions of $(a, 2)$-QXor-SAT are coarse and that the probability of satisfiability is almost 0 when the number of variables is around 2 times the number of clauses.

A random graph from the $G(n, p)$ model has $n$ vertices and each pair of vertices is linked with probability $p$. In [10], Erdős and Rényi located the density of edges at which the first connected component with more than one cycle - called a complex component - appears. Using analytic tools, Janson, Knuth, Luczak and Pittel derived in [15] more information on the structure of a random graph or multigraph near the birth of complex components. Söderberg introduced in [18] a model of inhomogeneous random graph $₫$, extended by Bollobás, Janson and Riordan [6]. This model generalizes $G(n, p)$ in the following way: each vertex receives a type among a set of $q$ types, and the probability that a vertex of type $i$ and one of type $j$ are linked is the coefficient $(i, j)$ of a symmetric matrix $R$ of dimension $q \times q$. Among other results, they located the birth of the complex component. We combine here the accuracy of the approach of [15] with the generality of the inhomogeneous random graph model. We also enrich the model, adding a weight $\sigma$ for each connected component.

1.2. Our Work. Random Boolean formulas with two variables per clause can be modeled by random multigraphs. Observe that the critical density $1 / 2$, common in [16, 8] and [7, corresponds to an important change in the structure of the underlying graphs: as the number of edges reaches half the number of vertices, components more complex than trees or unicyclics start to appear in random graphs (see for instance [4, 15). Our main goal is to establish a general framework that allows precise descriptions of some of the phase transitions of random formulas with 2 variables per clause. Namely our results apply to (and generalize) those in [16, 8].

\footnotetext{
${ }^{1}$ We thank Annika Heckel for bringing to our notice the existence of this model.
} 
In particular, they give a more detailed picture of the transitions introduced in [7] for quantified formulas. To do so, we study a new model of colored and weighted random multigraphs, similar to the inhomogeneous random graph model [18] and to the multigraph process 15 . We then propose a detailed analysis (thought still very general) with the purpose of quantifying the probability of satisfiability of formulas before and inside the critical window of their phase transitions. Our work is based on generating functions and analytic methods.

The rest of the paper is organized as follows: in Section 2, we first present our model and derive the main theorem on the asymptotic number of inhomogeneous multigraphs before and near the birth of complex components. Then, in Section 3 , we show how to use this theorem to derive the phase transition of several tractable satisfiability problems, namely bipartitness, quantified 2-Xor-formulas and random $k$-colorings. Section 4 is dedicated to the proof of the main theorem: first we derive the generating functions of the vertex-colored and edge-weighted trees, unicyclic graphs, paths of trees and graphs with cubic kernel of fixed excess. They are then gathered to build general multigraphs.

\section{Model and Main Theorem}

We consider labelled multigraphs - loops and multiple edges are allowed - with colored vertices and weighted edges. Let $R$ be a symmetric $q \times q$ matrix with nonnegative coefficients and $\sigma$ a fixed positive constant. Let $\left\{c_{1}, \ldots, c_{q}\right\}$ be a set of $q$ distinct colors. A multigraph $G$ is a $(R, \sigma)$-multigraph if

- each vertex $v$ of $G$ is colored with color $c(v) \in\left\{c_{1}, \ldots, c_{q}\right\}$,

- each edge $\overline{v w}$ of $G$ is weighted with $R_{c(v), c(w)}$,

- a weight $\sigma$ is given to each connected component.

Following [3, 5, 11, 15, the compensation factor $\kappa(G)$ of a multigraph $G$ with set of vertices $V$ and set of edges $E$ is

$$
\kappa(G):=\prod_{v \in V} 2^{-m_{v, v}} \prod_{\frac{v w}{v w E}}\left(m_{v, w} !\right)^{-1}
$$

where $m_{v, w}$ is the number of edges binding $u$ to $v$ in $G$. Let us consider an ordered sequence of $m$ ordered couples of vertices $\left(v_{1}, w_{1}\right), \ldots,\left(v_{m}, w_{m}\right)$. Interpreting each couple as an edge, this sequence describes a multigraph. The number of such sequences corresponding to a given multigraph $G$ with $m$ edges is exactly $2^{m} m ! \kappa(G)$. Therefore, the two following random processes induce the same probability distribution on the multigraphs with $n$ vertices and $m$ edges:

- draw among all multigraphs with $n$ vertices and $m$ edges with probability proportional to the compensation factors,

- draw uniformly and independently $2 m$ vertices in $[1, n]$ to form a sequence of couples $\left(v_{1}, w_{1}\right), \ldots,\left(v_{m}, w_{m}\right)$, output the corresponding multigraph.

The compensation factor is equal to 1 if and only if the multigraph contains neither loops nor multiple edges (such a multigraph is called simple). The sum of the compensation factors of all multigraphs with $n$ vertices and $m$ edges is called, for simplicity, their number and is equal to $\frac{n^{2 m}}{2^{m} m !}$ (which needs not be an integer). Given an $(R, \sigma)$-multigraph $G$, we define its weight $\omega(G)$ as the product of three terms: the compensation factor $\kappa(G)$, a factor $\sigma$ for each connected component and 
the product of the weights of its edges

$$
\omega(G)=\kappa(G) \times \sigma^{\mathrm{cc}(G)} \times \prod_{\overline{v w} \in E} R_{c(v), c(w)},
$$

where $\operatorname{cc}(G)$ is the number of connected components of $G$ and $E$ its set of edges.

There are three differences between our model and the original one, introduced by Söderberg [18. First, the number of edges is a parameter of the model, while in [18] each pair of vertices is linked by an edge with some probability. This is the same difference as between the classic graph models $G(n, m)$ and $G(n, p)$. Secondly, we consider multigraphs instead of simple graphs. Thirdly, the parameter $\sigma$ is new. It brings to the model the expressiveness needed to encode the constraint satisfaction problems considered in Section 3 .

An edge-weighted multigraph is vertex-transitive if its automorphism group is transitive and also preserve the weights - see for instance Godsil and Royle [14]. Intuitively, this means that, using only the topology of the multigraph, no vertex can be distinguished from another. Let $G$ be a multigraph with $q$ vertices and weighted edges. The weighted adjacency matrix $R$ of $G$ is a $q \times q$ matrix with entry $R_{i, j}$ equal to the sum of the weights of the edges between vertex $i$ and vertex $j$. For simplicity, we say that a matrix $R$ is vertex-transitive if it is symmetric, has nonnegative coefficients and the weighted multigraph associated is vertex-transitive. The special structure of those matrices implies several properties, in particular of their spectrum, which are listed in Lemma6. Many models using $(R, \sigma)$-multigraphs involve vertex-transitive matrices $R$, e.g. the 2-colorability and the quantified 2-Xor satisfiability problems, as will be shown in Section 3. Since our aim is to emphasize the link between the birth of complex components and the phase transition of some satisfiability or constraint problems, we focus on the case where $R$ is vertextransitive.

We define $g_{R, \sigma}(n, m)$ as the sum of the weights of the $(R, \sigma)$-multigraphs built with $n$ vertices and $m$ edges

$$
g_{R, \sigma}(n, m)=\sum_{|G|=n,\|G\|=m} \omega(G) .
$$

Theorem 1. Let $R$ be a $q \times q$ vertex-transitive matrix with greatest eigenvalue $\delta, \sigma$ a positive fixed constant, $c$ the number of connected components in the multigraph associated to $R$ and let $\chi(X)$ denote the polynomial $\prod_{\lambda \in \operatorname{Sp}(R) \backslash \delta}\left(1-\frac{\lambda}{\delta} X\right)$, where $\operatorname{Sp}(R)$ is the spectrum of $R$. For any $m / n$ in a closed interval of $] 0,1 / 2[$,

$$
g_{R, \sigma}(n, m) \sim \frac{n^{2 m}}{2^{m} m !}\left(1-\frac{2 m}{n}\right)^{\frac{1-c \sigma}{2}} \frac{\delta^{m}(\sigma q)^{n-m}}{\chi\left(\frac{2 m}{n}\right)^{\sigma / 2}} .
$$

As $n$ is large and $m=\frac{n}{2}\left(1+\mu n^{-1 / 3}\right)$ with $|\mu| \leq n^{1 / 12}$,

$$
g_{R, \sigma}(n, m) \sim \frac{n^{2 m}}{2^{m} m !} \phi_{c \sigma}(\mu) n^{(c \sigma-1) / 6} \frac{\delta^{m}(\sigma q)^{n-m}}{\chi(1)^{\sigma / 2}}
$$

where $\phi_{\sigma}(\mu)$ is equal to $\sqrt{2 \pi} \sum_{k} e_{k}^{(\sigma)} \sigma^{k} A\left(3 k+\frac{\sigma}{2}, \mu\right), e_{k}^{(\sigma)}$ is the $(2 k)$-th coefficient of $\left(\sum_{n \geq 0} \frac{(6 n) ! z^{2 n}}{(2 n) !(3 n) ! 2^{n}(3 !)^{n}}\right)^{\sigma}$ and $A(y, \mu)=\frac{e^{-\mu^{3} / 6}}{3(y+1) / 3} \sum_{k \geq 0} \frac{\left(3^{2 / 3} \mu / 2\right)^{k}}{k ! \Gamma((y+1-2 k) / 3)}$.

\section{Remarks.}


(1) As a corollary of Lemma 6, the number $c$ of connected components in the graph with adjacency matrix $R$ is equal to the multiplicity of the greatest eigenvalue $\delta$ of this vertex-transitive matrix.

(2) The polynomial

$$
\chi(X)=\prod_{\lambda \in \operatorname{Sp}(R) \backslash \delta}\left(1-\frac{\lambda}{\delta} X\right)
$$

is linked to the characteristic polynomial $P_{R}(X)=\operatorname{det}(X I-R)$ by the relation

$$
\chi(X)=\left(\frac{X}{\delta}\right)^{q} \frac{P_{R}\left(\frac{\delta}{X}\right)}{(1-X)^{c}} .
$$

(3) Since $R$ is a symmetric matrix with dominant eigenvalue $\delta$, all the values in $\operatorname{Sp}(R) \backslash \delta$ are real and smaller that $\delta$. Therefore, $\chi(1)$ is positive.

\section{Applications}

To describe the phase transition of a problem, we perform the following steps:

(1) build $R$ and $\sigma$ in order to obtain a one-to-one mapping between the instances of the problem and the $(R, \sigma)$-multigraphs,

(2) derive from $R$ the values $q, c, \delta, \chi\left(\frac{2 m}{n}\right)$ and $\chi(1)$ defined in Theorem 1

(3) apply Theorem 1

In the following section, we rediscover some results from Pittel and Yeum [16], prove new results on the satisfiability of quantified 2-Xor-formulas [7] and rederive the probability that a random $k$-coloring is proper [19.

3.1. Bipartite Multigraphs. A proper 2-coloring of a multigraph is a way of coloring the vertices with 2 colors such that no two adjacent vertices share the same one. A graph is bipartite if it admits a proper 2-coloring. In particular, such a graph contains no loop. In [16, the authors computed the probability for a random graph with $n$ vertices and $m$ edges to be bipartite, and we propose a new proof of some of their results.

Let $G$ be a multigraph with $n$ vertices and $c$ a function from $[1, n]$ to $\{1,2\}$. We define the vertex-colored and edge-weighted multigraph $G_{c}$ as follows: a color $c(v)$ is assigned to each vertex $v$, each edge $\overline{v w}$ has weight 1 if $c(v) \neq c(w)$ and 0 if $c(v)=c(w)$. The weight $\omega\left(G_{c}\right)$ of $G_{c}$ is $\kappa(G)$ times the product of the weights of the edges. Therefore, $\omega\left(G_{c}\right)=0$ if and only if there exist adjacent vertices $v$ and $w$ such that $c(v)=c(w)$. It follows that $\sum_{c:[1, n] \rightarrow\{1,2\}} \omega\left(G_{c}\right)$ is the number of ways to properly 2-color $G$. We just described a one-to-one mapping between the properly 2-colored multigraphs and the $\left(\left(\begin{array}{ll}0 & 1 \\ 1 & 0\end{array}\right), 1\right)$-multigraphs.

Every bipartite multigraph $G$ admits $2^{\operatorname{cc}(G)}$ proper 2-colorings, because such a coloring is characterized by the choice of one color in each connected component. Therefore, to count each bipartite multigraph exactly one time, each connected component receives a compensation factor $\frac{1}{2}$. This proves that the bipartite multigraphs are in a one-to-one mapping with the $\left(\left(\begin{array}{ll}0 & 1 \\ 1 & 0\end{array}\right), \frac{1}{2}\right)$-multigraphs.

For $R=\left(\begin{array}{ll}0 & 1 \\ 1 & 0\end{array}\right)$, we have $\delta=1, \chi(X)=1+X, q=2, c=1$ and $\sigma=\frac{1}{2}$. As a corollary of Theorem 1, we thus have

Theorem 2. Let $\mathbb{P}_{\mathrm{Bip}}(n, m)$ denote the probability that a random graph or multigraph with $n$ vertices and $m$ edges is bipartite, then 
- when $m / n$ is in a closed interval of $] 0,1 / 2[$,

$$
\mathbb{P}_{\text {Bip }}(n, m) \sim\left(\frac{1-\frac{2 m}{n}}{1+\frac{2 m}{n}}\right)^{1 / 4},
$$

- for any $|\mu| \leq n^{1 / 12}$,

$$
\lim _{n \rightarrow \infty} n^{1 / 12} \mathbb{P}_{\text {Bip }}\left(n, \frac{n}{2}\left(1+\mu n^{-1 / 3}\right)\right)=\phi_{1 / 2}(\mu),
$$

where $\phi_{1 / 2}(\mu)$, defined in Theorem 1 , decreases from 1 to 0 for $\mu$ in $\mathbb{R}$.

3.2. Quantified 2-Xor Formulas. In [7, the authors analyze quantified 2-Xor formulas. Those are quantified conjunctions of $m$ Xor-clauses with $\beta$ universal and $n$ existential variables

$$
\forall x_{1} \ldots x_{\beta} \exists y_{1} \ldots y_{n} \bigwedge_{i=1}^{m}\left(y_{f_{i, 1}} \oplus y_{f_{i, 2}}=\left(e_{i, 1} \wedge x_{1}\right) \oplus \cdots \oplus\left(e_{i, \beta} \wedge x_{\beta}\right)\right) .
$$

The values of the variables $\left(x_{i}\right)$ and $\left(y_{j}\right)$ can be considered equally as Booleans or bits, by identifying True to 1 and False to 0. The Boolean operator Xor $x \oplus y$ corresponds to the bit sum $(x+y \bmod 2)$, and the And Boolean operator $x \wedge y$ to the product $(x y \bmod 2)$. The authors study how the probability of satisfiability evolves with the number $m$ of clauses when the number $n$ of existential variables is large, and locate the value of $m$ at which the phase transition occurs.

Each clause

$$
y_{f_{i, 1}} \oplus y_{f_{i, 2}}=\left(e_{i, 1} \wedge x_{1}\right) \oplus \cdots \oplus\left(e_{i, \beta} \wedge x_{\beta}\right)
$$

is characterized by a triplet $\left(f_{i, 1}, f_{i, 2}, e_{i}\right)$ where $f_{i, 1}$ and $f_{i, 2}$ are integers in $[1, n]$ and $e_{i}$ is a $\beta$-tuple of bits. We consider clauses such that $e_{i}$ belongs to a fixed multiset $E$ of $\beta$-tuples of bits. We call the formulas that contain only those clauses the E-formulas. For example, $y_{1} \oplus y_{2}=x_{1} \oplus x_{3}$ is a $\{(1,0,1),(1,1,0)\}$-formula (with only one clause), but $y_{1} \oplus y_{2}=x_{2} \oplus x_{3}$ is not. For any integer $j$ in $\left[1,2^{\beta}\right]$, $[j]_{2}$ is the $\beta$-tuple of bits matching the binary decomposition of $j-1$

$$
[j]_{2}=\left(b_{0}, \ldots, b_{\beta-1}\right) \text { if and only if } j-1=\sum_{k=0}^{\beta-1} b_{k} 2^{k} .
$$

To a multiset $E$ of $\beta$-tuples of bits, we associate a matrix $R^{(E)}$ of dimension $2^{\beta} \times 2^{\beta}$ such that $R_{i, j}^{(E)}$ is the number of occurrences of $[i]_{2} \oplus[j]_{2}$ in $E$ :

$$
R_{i, j}^{(E)}=\#\left\{e \in E \mid[i]_{2} \oplus[j]_{2}=e\right\} .
$$

For example, when $\beta=2$ and $E=\left\{\left(\begin{array}{ll}0 & 1\end{array}\right),\left(\begin{array}{ll}1 & 0\end{array}\right)\right\}$, we have $R^{(E)}=\left(\begin{array}{llll}0 & 1 & 1 & 0 \\ 1 & 0 & 0 & 1 \\ 1 & 0 & 0 & 1 \\ 0 & 1 & 1 & 0\end{array}\right)$.

Lemma 3. Let $E$ be a multiset of $\beta$-tuples of bits. There exists a one-to-one mapping between

- the satisfiable E-formulas with $n$ existential variables and $m$ clauses,

- the $\left(R^{(E)}, 2^{-\beta}\right)$-multigraphs with $n$ vertices and $m$ edges.

Proof. Let $\phi$ denote the formula of Equation (3) and assume it is an $E$-formula. A solution of $\phi$ is a set $\eta_{1}, \ldots, \eta_{n}$ of $n \beta$-tuples of bits such that for each instantiation 
of the variables $x_{1}, \ldots, x_{\beta}$, the values

$$
\begin{gathered}
y_{1}=\eta_{1,1} x_{1} \oplus \cdots \oplus \eta_{1, \beta} x_{\beta}, \\
\vdots \\
y_{n}=\eta_{n, 1} x_{1} \oplus \cdots \oplus \eta_{n, \beta} x_{\beta}
\end{gathered}
$$

satisfy $\phi$. For example, the formula $\forall x_{1}, x_{2} \exists y_{1}, y_{2}, y_{1} \oplus y_{2}=x_{1}$ has 4 solutions $\left\{\left(\begin{array}{ll}1 & 0\end{array}\right),\left(\begin{array}{ll}0 & 0\end{array}\right)\right\},\left\{\left(\begin{array}{ll}1 & 1\end{array}\right),\left(\begin{array}{ll}0 & 1\end{array}\right)\right\},\left\{\left(\begin{array}{ll}0 & 0\end{array}\right),\left(\begin{array}{ll}1 & 0\end{array}\right)\right\}$, and $\left\{\left(\begin{array}{lll}0 & 1\end{array}\right),\left(\begin{array}{ll}1 & 1\end{array}\right)\right\}$. The first one matches the obvious solution $y_{1}=x_{1}, y_{2}=0$.

We first build a bijection between the couples ( $E$-formula, solution) and the $\left(R^{(E)}, 1\right)$-multigraphs. Each existential variable $y_{i}$ matches a vertex of color $\eta_{i}$, and each clause an edge. The number of couples ( $E$-formula, solution) is

$$
\sum_{\phi \in E \text {-formula }} \sum_{\text {solution of } \phi} 1 \text {. }
$$

The proof consists in switching the sums, assigning to each $y_{i}$ a linear combination

$$
y_{i}=\eta_{i, 1} x_{1} \oplus \cdots \oplus \eta_{i, \beta} x_{\beta},
$$

and to count the number of $E$-formulas satisfied by those $\left(y_{i}\right)$. By definition of $R^{(E)}$, this is equal to $g_{R^{(E)}, 1}(n, m)$.

To end the proof, we show that a satisfiable $E$-formula admits $2^{\beta \text { cc }}$ solutions, where $\mathrm{cc}$ is the number of connected components in its graph representation. Indeed, once the color (i.e. the $\beta$-tuple) of an existential variable is chosen, a transversal of the graph determines the colors of the other existential variables of the component. So we have exactly one choice of color for each connected component, and $2^{\beta}$ choices for this color.

This application is an opportunity to present some tools for deriving the parameters $c$ and $\chi(X)$ of Theorem 1 for non-trivial matrices $R$. Let $\operatorname{Ham}(\beta)$ denote the $2^{\beta} \times 2^{\beta}$ matrix defined by

$$
\operatorname{Ham}(\beta)_{i, j}= \begin{cases}1 & \text { if the Hamming distance between }[i]_{2} \text { and }[j]_{2} \text { is } 1, \\ 0 & \text { otherwise. }\end{cases}
$$

Xor-Clauses with one Universal Variable. If the Xor-clauses contain exactly one universal variable, like $y_{i} \oplus y_{j}=x_{k}$, then $E$ is the set $\left\{e_{1}, \ldots, e_{\beta}\right\}$, where, for all $i, e_{i}$ denotes the $\beta$-tuple of bits with a 1 at position $i$ and 0 elsewhere, and $R^{(E)}=\operatorname{Ham}(\beta)$. In this case, the number of colors is $q=\sigma^{-1}=2^{\beta}$. The matrix $\operatorname{Ham}(\beta)$ is irreducible, so $c=1$. Its greatest eigenvalue $\delta=\beta$ corresponds to the eigenvector $\overrightarrow{1}=\left(\begin{array}{lll}1 & \cdots & 1\end{array}\right)^{T}$. The matrix $\operatorname{Ham}(\beta)$ admits the following block decomposition 2 :

$$
\operatorname{Ham}(\beta+1)=\left(\begin{array}{cc}
\operatorname{Ham}(\beta) & I \\
I & \operatorname{Ham}(\beta)
\end{array}\right)
$$

so its characteristic polynomial is solution of the following recursive formula:

$$
P_{\operatorname{Ham}(\beta+1)}(X)=\operatorname{det}\left((X I-\operatorname{Ham}(\beta))^{2}-I^{2}\right)=P_{\operatorname{Ham}(\beta)}(X-1) P_{\operatorname{Ham}(\beta)}(X+1) .
$$

By induction,

$$
P_{\operatorname{Ham}(\beta)}(X)=\prod_{i=0}^{\beta}(X-(\beta-2 i))^{\left(\begin{array}{c}
\beta \\
i
\end{array}\right)}
$$

\footnotetext{
${ }^{2}$ We thank Timo Jolivet who helped us find this recursion.
} 
and, using Remark 2 ,

$$
\chi(X)=\prod_{i=1}^{\beta}\left(1-X\left(1-\frac{2 i}{\beta}\right)\right)^{\left(\begin{array}{c}
\beta \\
i
\end{array}\right)} .
$$

Xor-Clauses with $\alpha$ Universal Variables. We consider Xor-clauses that contain the ordered sum of exactly $\alpha$ universal variables, e.g. for $\alpha=3, y_{1} \oplus y_{2}=x_{1} \oplus x_{3} \oplus x_{1}$. In this case, $R^{(E)}=\operatorname{Ham}(\beta)^{\alpha}$. The parameters of this matrix are derived from those of $\operatorname{Ham}(\beta)$. The size $q=2^{\beta}$ is the same. The eigenvalues of $\operatorname{Ham}(\beta)^{\alpha}$ are those of $\operatorname{Ham}(\beta)$, raised at the power $\alpha$. In particular, the greatest eigenvalue of $\operatorname{Ham}(\beta)^{\alpha}$ is $\delta=\beta^{\alpha}$. If $\alpha$ is odd, $\operatorname{Ham}(\beta)^{\alpha}$ is irreducible and $c=1$. If $\alpha$ is even, the greatest eigenvalue of $\operatorname{Ham}(\beta)^{\alpha}$ has multiplicity 2, so $c=2$. Finally,

$$
\chi(X)=\prod_{i=1}^{\beta+1-c}\left(1-X\left(1-\frac{2 i}{\beta}\right)^{\alpha}\right)^{\left(\begin{array}{c}
\beta \\
i
\end{array}\right)} .
$$

Xor-Clauses with Distinct Universal Variables. If, furthermore, the $\alpha$ universal variables in each Xor-clause are constrained to be distinct, then $E$ is the set $\left\{e \in\{0,1\}^{\beta} \mid e_{1}+\cdots+e_{\beta}=\alpha\right\}$. Let $\operatorname{Ham}(\alpha, \beta)$ denote the matrix $R^{(E)}$. We claim that this matrix satisfies the recursive relation for all $0 \leq \alpha \leq \beta-2$

$$
\operatorname{Ham}(\beta) \operatorname{Ham}(\alpha+1, \beta)=(\beta-\alpha) \operatorname{Ham}(\alpha, \beta)+(\alpha+2) \operatorname{Ham}(\alpha+2, \beta) .
$$

Indeed, the coefficient $(i, j)$ of the matrix $\operatorname{Ham}(\beta) \operatorname{Ham}(\alpha+1, \beta)$ is the number of ways to write the bit-to-bit sum

$$
[i]_{2} \oplus[j]_{2}=e \oplus v_{\alpha+1}
$$

where $e$ and $v_{\alpha+1}$ are $\beta$-tuples of bits that contains respectively exactly $\alpha+1$ and 1 ones. There are now three cases.

- If $[i]_{2} \oplus[j]_{2}$ contains $\alpha$ ones, then $e$ has canceled a bit from $v_{\alpha+1}$. There are then $(\beta-\alpha)$ couples $\left(v_{\alpha+1}, i\right)$ which are solutions of Equation (4).

- If $[i]_{2} \oplus[j]_{2}$ contains $\alpha+2$ ones, then the one in $e$ is added to a zero of $v_{\alpha+1}$, and Equation (4) admits $\alpha+2$ solutions.

- Otherwise, Equation (4) has no solution.

Thus we can write

$$
R^{(E)}=P_{\alpha, \beta}(\operatorname{Ham}(\beta))
$$

where the polynomial $P_{\alpha, \beta}$ is characterized by the recursive formula $P_{0, \beta}(X)=1$, $P_{1, \beta}(X)=X$ and for all $\alpha$ in $[0, \beta-2]$, then

$$
(\alpha+2) P_{\alpha+2, \beta}(X)=X P_{\alpha+1, \beta}(X)-(\beta-\alpha) P_{\alpha, \beta}(X) .
$$

Again, the parameters of $R^{(E)}$ are derived from those of $\operatorname{Ham}(\beta)$. Observe that $P_{\alpha, \beta}(\beta)=$ $\left(\begin{array}{c}\beta \\ \alpha\end{array}\right)$ and $P_{\alpha, \beta}(-\beta)=(-1)^{\alpha}\left(\begin{array}{l}\beta \\ \alpha\end{array}\right)$. The size is $q=2^{\beta}$, and the greatest eigenvalue is $\delta=P_{\alpha, \beta}(\beta)=\left(\begin{array}{c}\beta \\ \alpha\end{array}\right)$. If $\alpha$ is odd, $\operatorname{Ham}(\beta)^{\alpha}$ is irreducible and $c=1$, else $c=2$.

Finally

$$
\chi(X)=\prod_{i=1}^{\beta+1-c}\left(1-X \frac{P_{\alpha, \beta}(\beta-2 i)}{\left(\begin{array}{l}
\beta \\
\alpha
\end{array}\right)}\right)^{\left(\begin{array}{c}
\beta \\
i
\end{array}\right)}
$$


Xor-Clauses with $\alpha$ Universal Variables and a Constant Term. We consider Xorclauses of the form

$$
y_{i} \oplus y_{j}=e_{1} x_{1} \oplus \cdots \oplus e_{\beta} x_{\beta} \oplus e_{\beta+1}
$$

where exactly $\alpha$ of the bits $e_{1}, \ldots, e_{\beta}$ are 1 's. The set $E$ contains now $(\beta+1)$ tuples of bit. To take the term $e_{\beta+1}$ into account, any solution of such a formula now assigns to each existential variable $y_{i}$ a affine combination of universal variables plus a constant 0 or 1 , so

$$
y_{i}=\eta_{i, 1} x_{1} \oplus \cdots \oplus \eta_{i, \beta} x_{\beta} \oplus \eta_{i, \beta+1} .
$$

where $\eta_{i}$ is a $(\beta+1)$-tuple of bits. Let $E_{\alpha}$ denote the set $E$ corresponding to Xorclauses with $\alpha$ universal variables, and $E_{\alpha, \epsilon}$ the corresponding set with the option of adding a constant. Each $\beta$-tuple $e$ in $E_{\alpha}$ matches two $(\beta+1)$-tuples in $E_{\alpha, \epsilon}$ : both with the same first $\beta$ bits as $e$, one with last bit 0 , and the other with last bit 1 . Therefore, the matrix $R^{\left(E_{\alpha, \epsilon}\right)}$ is equal to

$$
\left(\begin{array}{ll}
R^{\left(E_{\alpha}\right)} & R^{\left(E_{\alpha}\right)} \\
R^{\left(E_{\alpha}\right)} & R^{\left(E_{\alpha}\right)}
\end{array}\right) \text {. }
$$

The spectrum of $R^{\left(E_{\alpha, \epsilon}\right)}$ is the same as the one of $R^{\left(E_{\alpha}\right)}$, except that each eigenvalue is doubled and the eigenvalue 0 is added with multiplicity $2^{\beta}$. We then obtain the parameters $q=1 / \sigma=2^{\beta+1}, c=1$ if $\alpha$ is odd and $c=0$ otherwise, $\delta=2 \beta^{\alpha}$ and $\chi(X)$ as in the following theorem. Injecting those parameters into Theorem 1 and dividing by the total number of $E_{\alpha, \epsilon}$-formulas

$$
\frac{n^{2 m}}{2^{m} m !}\left(2 \beta^{\alpha}\right)^{m}
$$

gives the following result.

Theorem 4. Let us consider a random quantified 2-Xor formula of the form (3) with $n$ existential variables, $\beta$ universal variables and $m$ Xor-clauses containing two existential variables, $\alpha$ universal variables and one constant term in $\{0,1\}$. Let $\mathbb{P}_{\mathrm{SAT}}$ denote the probability that such a formula is satisfiable, $c=2$ if $\alpha$ is even, $c=1$ otherwise, and

$$
\chi(X)=\prod_{i=1}^{\beta+1-c}\left(1-2(1-X 2 i / \beta)^{\alpha}\right)^{\left(\begin{array}{c}
\beta \\
i
\end{array}\right)},
$$

then

- as $n$ is large and $m / n$ is restrained to a closed interval of $] 0,1 / 2[$,

$$
\mathrm{P}_{\mathrm{SAT}} \sim \chi(2 m / n)^{-2^{-\beta-2}}(1-2 m / n)^{\frac{1}{2}-c 2^{-\beta-2}}
$$

- for any fixed real value $x$ and $m=\frac{n}{2}\left(1+\mu n^{-1 / 3}\right)$,

$$
\lim _{n \rightarrow \infty} n^{\left(1-c 2^{-\beta-1}\right) / 6} \mathrm{P}_{\mathrm{SAT}}=\chi(1)^{-2^{-\beta-2}} \phi_{c 2^{-\beta-1}}(\mu),
$$

where $\phi_{\sigma}(\mu)$ is a computable function defined in Theorem 1 .

Combining the previous results, we could as well consider quantified 2-Xor formulas with $\alpha$ distinct universal variables and a constant term in each clause. 
3.3. Random $k$-Coloring of Random Multigraphs. The following theorem, due to Wright [19, enumerates the properly $q$-colored multigraphs. We propose a new proof using the formalism of $(R, \sigma)$-multigraphs.

Theorem 5. If $m / n$ is fixed in ]0,1/2[, the asymptotic probability that a random $q$-coloring of a random multigraph with $n$ vertices and $m$ edges is proper is

$$
\left(1-\frac{1}{q}\right)^{m}\left(1+\frac{1}{q-1} \frac{2 m}{n}\right)^{-\frac{q-1}{2}} .
$$

Proof. A multigraph properly $q$-colored is a $(R, 1)$-multigraph where $R_{i, j}=1$ for all $i \neq j$ and 0 otherwise. Their asymptotics is derived from Theorem 11 with the parameters $c=1, \chi(X)=(1+X /(q-1))^{q-1}$. It is then divided by the total number of multigraphs with $n$ vertices and $m$ edges randomly (and possibly not properly) $q$-colored, which is $\frac{n^{2 m}}{2^{m} m !} q^{n}$.

In fact, this result holds for any positive fixed value of $m / n$ [19]. There is a proof of this result in the setting of inhomogeneous multigraphs, which is an interesting development that will be part of a forthcoming publication. Here, we just sketch this proof. It starts with the direct expression

$$
g_{R, 1}(n, m)=\frac{1}{2^{m} m !} \sum_{\substack{\vec{n} \in \mathbb{N}^{q} \\
\stackrel{n}{n}=n}}\left(\begin{array}{c}
n \\
n_{1}, \ldots, n_{q}
\end{array}\right)(\overleftarrow{n} R \vec{n})^{m}
$$

We then apply Theorem 5.4.8 of [17] to conclude. This approach can be generalized to any irreducible aperiodic matrix $R$ when $\sigma=1$.

Theorem [ 5 is not to be confused with an asymptotic of $q$-colorable multigraphs, because a colorable multigraph may have several proper colorings.

\section{Proof of Theorem 1}

4.1. Properties of Vertex-Transitive Matrices. The notation $\overrightarrow{1}$ stands for the column vector with all coefficients equal to 1 . The structure of a vertex-transitive matrix implies the following properties:

Lemma 6. Let $R$ denote a $q \times q$ vertex-transitive matrix with non-negative coeffcients, then there exist

(1) an integer $c$, a permutation matrix $P$ and a square matrix $S$ of dimension $\frac{q}{c} \times \frac{q}{c}$ such that $P R P^{-1}=\operatorname{diag}(S, \ldots, S)$, where the block diagonal matrix contains $c$ blocks,

(2) a positive $\delta$, eigenvalue of $S$ of multiplicity 1 , such that $S \overrightarrow{1}=\delta \overrightarrow{1}$ and for all $\lambda$ in the spectrum of $S,|\lambda| \leq \delta$,

(3) an orthogonal matrix $Q$ and a diagonal matrix $\Delta$ such that $S=Q \Delta Q^{T}$, $\Delta_{1,1}=\delta$ and $Q \overrightarrow{e_{1}}=\sqrt{\frac{c}{q}} \overrightarrow{1}$.

Proof. Because the multigraph $G$ associated to $R$ is vertex-transitive, all pairs of connected components are isomorphic. The matrix $S$ denotes the weighted adjacency matrix of one of those components and $c$ is their number. In an edge-weighted multigraph, the degree of a vertex $v$ is the sum of the weights of the edges that contain $v$. All the degrees in $G$ are equal, otherwise two vertices with different degrees could be distinguish, so $\overrightarrow{1}$ is an eigenvector of $R$ and $\delta$ denotes this common degree. 
Applying the Perron-Fröbenius Theorem, we conclude that the eigenvalue $\delta$ has multiplicity 1 and is greater or equal in absolute value than any other eigenvalue. Finally, real symmetric matrices are diagonalizable by orthogonal matrices.

Let $\delta$ denote the greatest real eigenvalue of $R$. We can assume it to be equal to 1 without loss of generality, replacing $R$ by $\frac{1}{\delta} R$ and $g(n, m)$ by $\delta^{m} g(n, m)$. We can also assume that the number $c$ of connected components of the multigraph encoded by $R$ is 1 : the $(R, \sigma)$-multigraphs are in a one-to-one mapping with the $(S, c \sigma)$ multigraphs where $S$ is the adjacency matrix of one of the connected components. In the rest of this section, $R$ is assumed to be a $q \times q$ irreducible vertex-transitive matrix with greatest eigenvalue 1 .

4.2. Trees and Unicyclic Components. In [15], graphs are decomposed in three parts: trees, unicyclic components and complex components 20]. Their generating functions are expressed in term of the Cayley tree function $T(z)$ that counts the rooted labelled trees and is characterized by the equation $T(z)=z e^{T(z)}$. We follow the same approach.

An $R$-tree is a connected $R$-multigraph without cycle. If one vertex is marked, we say that the tree is rooted. A connected $R$-multigraph with exactly one cycle is called an $R$-unicyclic multigraph. Let $T_{i}(z), U(z)$ and $V(z)$ denote the generating functions of $R$-rooted trees with root of color $i$, unrooted trees and unicyclic multigraphs. Let also $\vec{T}(z)$ denote the vector $\left(T_{1}(z) \cdots T_{q}(z)\right)^{T}$. A $R$-path of trees is a colored directed path that links two vertices (that may not be distinct) of color $i$ and $j$, and each internal vertex of the path is the root of a colored $R$-rooted tree. Its generating function is denoted by $P_{i, j}(z)$.

Lemma 7. If $R$ is irreducible with greatest eigenvalue 1 , the generating functions of $R$-rooted trees, unrooted trees, unicyclic graphs and paths of trees are

$\vec{T}(z)=T(z) \overrightarrow{1}$

$$
\begin{array}{r}
V(z)=-\frac{1}{2} \log (1-T(z))-\frac{1}{2} \log (\chi(T(z))) \\
P_{i, j}(z)=\frac{1}{q(1-T(z))}+\sum_{l=2}^{q} Q_{i, l} Q_{j, l} \frac{\Delta_{l, l}}{1-\Delta_{l, l} T(z)}
\end{array}
$$$$
U(z)=q\left(T(z)-\frac{1}{2} T(z)^{2}\right)
$$

where $T(z)$ is the Cayley tree function and $R=Q \Delta Q^{T}$ as in Lemma 6 .

Proof. Using the analytic combinatorics tools (a good reference is [12]), the combinatorial specification of $R$-rooted trees translates into the following equations: for all $i, T_{i}(z)$ is equal to $z \exp \left(\operatorname{row}_{i}(R) \vec{T}(z)\right)$. Since $R$ is vertex-transitive, for all $i, j$, $T_{i}(z)=T_{j}(z)$, so $T_{i}(z)=\frac{1}{\delta} T(\delta z)$. an $R$-unrooted tree with a marked vertex is an $R$-rooted tree with root of unknown color, so $z U^{\prime}(z)=\sum_{i=1}^{q} T_{i}(z)$. Similarly, an $R$-unicyclic graph with a marked vertex on its cycle and an orientation is an $R$-path of rooted trees, so $u \partial_{u} V(z, u)=\frac{1}{2} \sum_{i=1}^{q} \sum_{k \geq 1}(u T(z) R)_{i, i}^{k}$ where $u$ marks the vertices of the cycle and $V(z)=V(z, 1)$. Finally, $P_{i, j}(z)=\left(R(I-T(z) R)^{-1}\right)_{i, j}$ and Lemma 6 lead to the announced expression.

Observe that at the first order, $U(z), V(z)$ and $P_{i, j}(z)$ are equal or proportional to their non-colored counterparts $T(z)-\frac{1}{2} T(z)^{2},-\frac{1}{2} \log (1-T(z))$ and $\frac{1}{1-T(z)}$. Furthermore, the first order of $P_{i, j}(z)$ is independent of $i$ and $j$.

We will prove in Theorem 9 that when $m / n<1 / 2$, almost all $(R, \sigma)$-multigraphs with $n$ vertices and $m$ edges contain only trees and unicyclic components. Theorem 8 is then equivalent to the first statement of Theorem 1 . 
Theorem 8. With the notations of Theorem 1, the number of $(R, \sigma)$-multigraphs that contain only trees and unicyclic components is

$$
g_{R, \sigma}^{(0)}(n, m) \sim \frac{n^{2 m}}{2^{m} m !}\left(1-\frac{2 m}{n}\right)^{\frac{1-\sigma}{2}} \frac{(q \sigma)^{n-m}}{\chi\left(\frac{2 m}{n}\right)^{\sigma / 2}} .
$$

Proof. A multigraph without complex component is a set of $n-m$ trees and of unicyclic components, so

$$
g_{R, \sigma}^{(0)}(n, m)=n !\left[z^{n}\right] \frac{(\sigma U(z))^{n-m}}{(n-m) !} e^{\sigma V(z)} .
$$

We then apply Theorem VIII.8 of [12, p.587] to derive the asymptotics of the coefficient extraction.

4.3. Complex Components. The notions of excess and kernel were first combined with a generating function approach in [20] and then [15]. This section relies on their work. The excess of a graph is defined as the difference between the number of edges and of vertices $k=m-n$. A component with excess $(-1)$ (resp. 0) is a tree (resp. unicyclic). The complex part of a multigraph is the set of its connected components that have positive excess. Deleting the vertices of degree one and merging the vertices of degree two, each graph can be reduced to a simpler graph, called its kernel, with minimum degree at least three. Reciprocally, any such graph can be developed by replacing edges by paths and adding trees to the vertices. The set $\mathbb{K}_{k}$ of kernels of excess $k$ is finite. Among them, the kernels that maximize the number of edges are the cubic (i.e. 3-regular) multigraphs $\mathbb{K}_{k}^{(3)}$. with $2 k$ vertices and $3 k$ edges. Their number, counted with their compensation factors and a weight $\sigma$ for each connected component, is computable

$$
\left|\mathbb{K}_{k, \sigma}^{(3)}\right|=\sum_{G \in \mathbb{K}_{k}^{(3)}} \kappa(G) \sigma^{\mathrm{cc}(G)}=(2 k) !\left[z^{2 k}\right]\left(\sum_{n \geq 0} \frac{(6 n) !}{(3 !)^{2 n} 2^{3 n}(3 n) !} \frac{z^{2 n}}{(2 n) !}\right)^{\sigma} .
$$

The generating function of complex (i.e. without trees and unicyclic components) $(R, \sigma)$-multigraphs of excess $k$ is

$$
K_{k, \sigma}(z)=\sum_{G \in \mathbb{K}_{k}} \sum_{\vec{c} \in[1, q]^{|G|}} \frac{\kappa(G) \sigma^{\operatorname{cc}(G)}}{|G| !} \prod_{i \in[1,|G|]} T_{c_{i}}(z) \prod_{(i, j) \in \operatorname{edge}(G)} P_{c_{i}, c_{j}}(z) .
$$

Since $\mathbb{K}_{k}$ is finite, this generating function is a rational function in $T(z)$. In its partial fraction decomposition, the term with denominator containing the highest power of $1-T(z)$ is

$$
\frac{\left|\mathbb{K}_{k, \sigma}^{(3)}\right|}{(2 k) ! q^{k}} \frac{T(z)^{2 k}}{(1-T(z))^{3 k}} .
$$

Theorem 9. When $m / n<1 / 2$ is fixed, almost all $(R, \sigma)$-multigraphs have an empty complex part.

Proof. In all the proof, $m / n<1 / 2$ is assumed to be fixed. A multigraph with $n$ vertices, $m$ edges and complex part of excess $k$ is a set of $n-m+k$ trees, a set of 
unicyclic components and a complex part. Therefore, the number of such $(R, \sigma)$ multigraphs is

$$
g_{R, \sigma}^{(k)}(n, m)=n !\left[z^{n}\right] \frac{(\sigma U(z))^{n-m+k}}{(n-m+k) !} e^{\sigma V(z)} K_{k, \sigma}(z) .
$$

With $g_{R, \sigma}^{(0)}(n, m)$ defined as in Theorem 8 , the theorem states that when $m / n<1 / 2$ is fixed,

Since

$$
g_{R, \sigma}(n, m) \sim g_{R, \sigma}^{(0)}(n, m) .
$$

$$
g_{R, \sigma}(n, m)=\sum_{k \geq 0} g_{R, \sigma}^{(k)}(n, m),
$$

this is equivalent with

$$
\lim _{n \rightarrow \infty} \sum_{k \geq 1} \frac{g_{R, \sigma}^{(k)}(n, m)}{g_{R, \sigma}^{(0)}(n, m)}=0 .
$$

It is well known that Theorem 9 holds for classic multigraphs. Therefore, if $g_{(1), 1}^{(k)}(n, m)$ denotes the sum of the compensation factors of multigraphs with $n$ vertices, $m$ edges and complex part of excess $k$, then $g_{(1), 1}^{(0)}(n, m)$ has the same asymptotics as the total number of multigraphs

$$
g_{(1), 1}^{(0)}(n, m) \sim \frac{n^{2 m}}{2^{m} m !} .
$$

Combined with Theorem 8 , this equivalence implies that there exists a constant $C_{1}$, which depends only on $m / n$, such that for $n$ large enough,

$$
g_{R, \sigma}^{(0)}(n, m) \geq C_{1}(q \sigma)^{n-m} g_{(1), 1}^{(0)}(n, m) .
$$

Since an $(R, \sigma)$-multigraph is a multigraph where each vertex has a color among a set of size $q$, each edge has a weight at most $r=\max _{i, j} R_{i, j}$ and each connected component a weight $\sigma$,

$$
g_{R, \sigma}^{(k)}(n, m) \leq q^{n} r^{m} \max (\sigma, 1)^{n} g_{(1), 1}^{(k)}(n, m) .
$$

Combining Equations (10) and (11), we conclude that there exist two constants $C_{2}$ and $C_{3}$, independent of $n$ and $k$, such that for $n$ large enough,

$$
\frac{g_{R, \sigma}^{(k)}(n, m)}{g_{R, \sigma}^{(0)}(n, m)} \leq C_{2}\left(C_{3} r^{m / n}\right)^{n} \frac{g_{(1), 1}^{(k)}(n, m)}{g_{(1), 1}^{(0)}(n, m)}
$$

where $r$ is the maximum of the coefficients of $R$.

Since Theorem 9 is equivalent with Equation (9) and holds for classic multigraphs, the previous inequality proves that

$$
\lim _{n} \sum_{k \geq 0} \frac{g_{R, \sigma}^{(k)}(n, m)}{g_{R, \sigma}^{(0)}(n, m)}=0
$$

as soon as $C_{3} r^{m / n}$ is smaller than 1, i.e. for matrices $R$ with small enough coefficients. But Theorem 9 is independent of the size of the coefficients of $R$, because this matrix can be replaced by $\alpha R$ for any positive $\alpha$ without changing the structure of the graphs. 
Theorem 10. With the notations of Theorem 1, when $m=\frac{n}{2}\left(1+\mu n^{-1 / 3}\right)$ and $|\mu| \leq$ $n^{1 / 12}$, the asymptotic number of $(R, \sigma)$-multigraphs with complex part of excess $k$ is

$$
g_{R, \sigma}^{(k)}(n, m) \sim \frac{n^{2 m}}{2^{m} m !} \frac{(\sigma q)^{n-m}}{\chi(1)^{\sigma / 2}} n^{(\sigma-1) / 6} \sigma^{k} e_{k}^{(\sigma)} \sqrt{2 \pi} A\left(3 k+\frac{\sigma}{2}, \mu\right) .
$$

Proof. In Equation (5), there are two saddle-points that are distinct when $m / n<$ $1 / 2$, but coalesce at this critical value. In this context, the large powers scheme ceases to apply, so we replace it with [15, Lemma 3] to obtain Equation (8) (see also [2, Theorem 11] and [12, Theorem IX.16] for links with the stable laws of probability theory). This lemma computes asymptotics of the shape

$$
\left[z^{n}\right] \frac{U(z)^{n-m}}{(1-T(z))^{y}}
$$

where $y$ is a real constant. In particular, it proves that for any real fixed real values $y_{1}$ and $y_{2}$,

$$
\left[z^{n}\right] \frac{U(z)^{n-m}}{(1-T(z))^{y_{1}}} \sim n^{\left(y_{1}-y_{2}\right) / 3}\left[z^{n}\right] \frac{U(z)^{n-m}}{(1-T(z))^{y_{2}}} .
$$

Therefore, in Equation (8), the only term of $K_{k, \sigma}(z)$ that influence the asymptotic is given by (7). We then apply Lemma 3 of [15] to

$$
g_{R, \sigma}^{(k)}(n, m) \sim \frac{(n-m) !}{(n-m+k) !} \frac{(\sigma q)^{n-m}}{\chi(1)^{\sigma / 2}} \frac{\sigma^{k}\left|\mathbb{K}_{k, \sigma}^{(3)}\right|}{2^{k}(2 k) !} \frac{n !}{(n-m) !}\left[z^{n}\right] \frac{\left(T(z)-\frac{T(z)^{2}}{2}\right)^{n-m}}{(1-T(z))^{3 k+\sigma / 2}} .
$$

Theorem 11 is then established by summation of the $g_{R, \sigma}^{(k)}(n, m)$. The result is multiplied by $\delta^{m}, \sigma$ is replaced by $c \sigma, q$ by $q / c$ and $\chi(X)$ is adjusted. More information on the analytic behavior of $A(y, \mu)$ can be found in [15, Lemma 3].

\section{Conclusion}

We have presented a model of random multigraphs with colored vertices and weighted edges, similar to the inhomogeneous graph model [18]. Using tools developed in [15], we derived a complete picture of the finite size scaling and the critical exponents associated to the birth of complex components. Applications to bipartite graphs and to the satisfiability of quantified 2-Xor-formulas raised new proof of known results [16] and new results.

In this paper, the emphasis is on the link between the birth of complex components in $(R, \sigma)$-multigraphs and the phase transition of tractable satisfiability problems. This justifies the restriction to vertex-transitive matrices $R$, often encountered in applications, and the addition of the factor $\sigma$ to the original inhomogeneous graph model in order to enrich the expressiveness.

The present results can be extended to simple $(R, \sigma)$-graphs. Indeed, almost surely, all loops and multiple edges of the random $(R, \sigma)$-multigraphs considered belong to unicyclic components. So the only adjustment needed is to replace the generating function $V(z)$ with $V(z)-\frac{1}{2} \sum_{i} R_{i, i} T_{i}(z)-\frac{1}{4} \sum_{i, j} R_{i, j}^{2} T_{i}(z) T_{j}(z)$. Due to the lack of space, the proof of this result is not included.

We now plan to extend our result to non-vertex-transitive matrices $R$, and to enumerate $R$-multigraphs with a larger density of edges. 


\section{REFERENCES}

1. Dimitris Achlioptas and Cris Moore, Random k-SAT: Two moments suffice to cross a sharp threshold, SIAM Journal of Computing 36 (2006), 740-762.

2. Cyril Banderier, Philippe Flajolet, Gilles Schaeffer, and Michèle Soria, Random maps, coalescing saddles, singularity analysis, and Airy phenomena, Random Structures Algorithms 19 (2001), no. 3-4, 194-246.

3. Béla Bollobás, A probabilistic proof of an asymptotic formula for the number of labelled regular graphs, European Journal of Combinatorics 1 (1980), 311-316.

4. _ Random graphs, Cambridge Studies in Advanced Mathematics, 1985.

5. Béla Bollobás and Alan M. Frieze, On matchings and hamiltonian cycles in random graphs, Random Graphs '83. Based on lectures presented at the 1st Poznań Seminar on Random Graphs (Michał Karoński and Andrzej Ruciński, eds.), North-Holland Mathematics Studies, vol. 118, North-Holland, 1985, pp. $23-46$.

6. Béla Bollobás, Svante Janson, and Oliver Riordan, The phase transition in inhomogeneous random graphs, Random Structures and Algorithms 31 (2007), no. 1, 3-122.

7. Nadia Creignou, Hervé Daudé, and Uwe Egly, Phase transition for random quantified XORformulas, Journal . Artif. Intell. Res. 29 (2007), 1-18.

8. Hervé Daudé and Vlady Ravelomanana, Random 2-XORSAT phase transition, Algorithmica 59(1) (2011), 48-65.

9. Olivier Dubois, R. Monasson, B. Selman, and R. Zecchina, Phase transition in combinatorial problems, vol. 265(1-2), Elsevier, 2001.

10. Paul Erdős and Alfréd Rényi, On the evolution of random graphs, publication of the mathematical institute of the hungarian academy of sciences 5 (1960), 17.

11. Philippe Flajolet, Donald E. Knuth, and Boris Pittel, The first cycles in an evolving graph, Discrete Mathematics 75 (1989), no. 1-3, 167-215.

12. Philippe Flajolet and Robert Sedgewick, Analytic combinatorics, Cambridge University Press, 2009.

13. Ehud Friedgut, Sharp thresholds of graph properties, and the $k$-sat problem, Journal of the A.M.S. 12(4) (1999), 1017-1054.

14. Chris Godsil and Gordon Royle, Algebraic graph theory, graduate texts in mathematics, NewYork : Springer-Verlag, 2001.

15. Svante Janson, Donald E. Knuth, Tomasz Luczak, and Boris Pittel, The birth of the giant component, Random Structures and Algorithms 4 (1993), no. 3, 233-358.

16. Boris Pittel and Ji-A Yeum, How frequently is a system of 2-linear boolean equations solvable?, Electronic Journal of Combinatorics 17 (2010).

17. Mark C. Wilson Robin Pemantle, Analytic combinatorics in several variables, Cambridge University Press, New York, NY, USA, 2013.

18. Bo Söderberg, General formalism for inhomogeneous random graphs, Phys. Rev. E 66 (2002), 066-121.

19. Edward M. Wright, Counting coloured graphs III, Canadian Journal of Mathematics 14 (1972), no. 1, 82-89.

20. $317-330$. 